\title{
15.3: Analytical Solutions for the Wide-View LCDs with Uniaxial-Film Compensation
}

\author{
Xinyu Zhu, Zhibing Ge, and Shin-Tson Wu \\ College of Optics and Photonics, University of Central Florida, Orlando, Florida 32816-2700
}

\begin{abstract}
Based on the Poincaré sphere representation, for the first time, we have derived the analytical solutions for the uniaxial-filmcompensated wide-view LCDs. Through the analyses of phase retardation of uniaxial-film, origin of dark-state light leakage, and spherical trigonometry, we are able to track the polarization state changes on the Poincaré sphere for any oblique incident light. These analysis procedures can be applied to optimize the viewing angle of uniaxial-film-compensated in-plane switching and multi-domain vertical alignment $L C D$ s.
\end{abstract}

\section{Introduction}

Wide-viewing-angle (WVA) is a critical requirement for high-end liquid crystal displays (LCDs). So far, two camps of WVA technologies have been developed: In-Plane Switching (IPS) [1] and Multi-domain Vertical Alignment (MVA) [2]. However, their intrinsic viewing angle is limited by the off-axis light leakage of the crossed polarizers. To suppress the light leakage at oblique angles, several phase compensation schemes using uniaxial films [3-6] and biaxial films [7-8] have been proposed. Computer simulation and experimental results have been reported, but the rigorous analytical solutions have not been published.

The objective of this paper is to provide comprehensive analytical solutions for the WVA LCDs with uniaxial-film compensation. With the analytical solutions, the inter-dependency between LC cell and film parameters can be clearly revealed. More importantly, analytical solutions provide a clear physical description about the compensation mechanisms. In this paper, we will first analyze the phase compensation principles using Poincaré sphere representation and then apply these principles to optimize the viewing angle of uniaxial-film-compensated IPS and MVA LCDs.

\section{Film compensation principles}

\subsection{Phase retardation of uniaxial media at oblique incidence}

In the uniaxial-film-compensated LCDs, both a-film and c-film are commonly used. For an a-film, its optic axis lies in the plane parallel to the film surface, and the phase retardation of the a-film at an arbitrary incident angle is given by:

$$
\begin{gathered}
\Gamma_{a}=\frac{2 \pi}{\lambda} d\left[n_{e} \sqrt{1-\frac{\sin ^{2} \theta_{0} \sin ^{2}\left(\phi_{n}-\phi_{0}\right)}{n_{e}^{2}}-\frac{\sin ^{2} \theta_{0} \cos ^{2}\left(\phi_{n}-\phi_{0}\right)}{n_{o}^{2}}}\right. \\
\left.-n_{o} \sqrt{1-\frac{\sin ^{2} \theta_{0}}{n_{o}^{2}}}\right] .
\end{gathered}
$$

While in the case of c-film, its optic axis is perpendicular to the film surface, and its phase retardation at any oblique incidence is

$$
\Gamma_{c}=\frac{2 \pi}{\lambda} n_{o} d\left(\sqrt{1-\frac{\sin ^{2} \theta_{0}}{n_{e}^{2}}}-\sqrt{1-\frac{\sin ^{2} \theta_{0}}{n_{o}^{2}}}\right) .
$$

2.2 Light leakage of crossed polarizers at oblique view Figure 1(a) shows the case when an oblique light traverses through two sheet polarizers. The polarizer's absorption axis $\mathbf{O M}$ makes an angle $\phi_{1}$ with respect to the $\mathrm{x}$-axis in the $\mathrm{x}$-y plane while the analyzer's absorption axis $\mathbf{O N}$ is oriented at angle $\phi_{2}$. The shadow triangle $\mathbf{O A B}$ denotes the incidence plane. The light beam, denoted by the wave vector $\mathbf{O K}$, propagates at azimuthal angle $\phi_{k}$ and polar angle $\theta_{k}$ inside the sheet polarizers. Although these two linear polarizers form an angle $\left(\phi_{2}-\phi_{1}\right)$ in the $\mathrm{x}$-y plane, their projections in the wave plane, however, form another angle $\angle \mathbf{M K N}$. We call this angle $\angle \mathbf{M K N}$ as the effective polarizer angle in the wave plane, which is expressed as $\varphi$ hereafter. The extinction ratio of the crossed polarizers depends on this effective polarizer angle $\varphi$ in the wave plane.

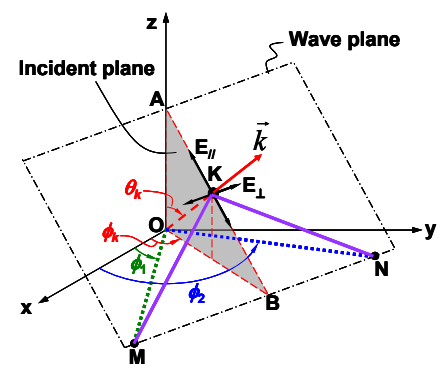

(a)

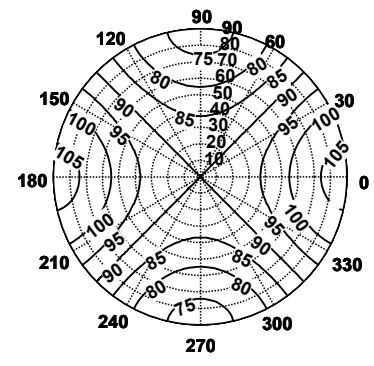

(b)
Fig. 1. (a) Schematic view of the effective polarizer angle $\varphi$ of two sheet polarizers in the wave plane of an oblique incident light, and (b) dependence of effective polarizer angle $\varphi$ on the azimuthal angle $\phi_{0}$ and polar angle $\theta_{0}$ with $\phi_{1}=45^{\circ}$ and $\phi_{2}=-45^{\circ}$, respectively.

Based on the dot product of vectors, If we set $\phi_{1}=45^{\circ}$ and $\phi_{2}=-45^{\circ}$, then the effective polarizer angle $\varphi$ can be expressed by:

$\varphi=\cos ^{-1}\left[\frac{-\sin ^{2} \theta_{k} \cos \left(\pi / 4-\phi_{k}\right) \cos \left(\pi / 4+\phi_{k}\right)}{\sqrt{1-\sin ^{2} \theta_{k} \cos ^{2}\left(\pi / 4-\phi_{k}\right)} \sqrt{1-\sin ^{2} \theta_{k} \cos ^{2}\left(\pi / 4+\phi_{k}\right)}}\right]$,

where $\phi_{k}=\phi_{0}$ and $\theta_{k}=\sin ^{-1}\left(\sin \theta_{0} / n_{p}\right)$. Here $n_{p}(\sim 1.5)$ is the average real refractive index of the sheet polarizer. Based on the above equation, Fig. 1(b) plots the dependence of effective polarizer angle $\varphi$ on viewing polar angle $\theta_{0}$ and azimuthal angle $\phi_{0}$. Apparently, when $\phi_{k}=\phi_{0}=0^{\circ}, 90^{\circ}, 180^{\circ}$, and $270^{\circ}$, the effective polarizer angle $\varphi$ reaches the extrema, which deviate the farthest from $90^{\circ}$. The effective polarizer angle deviating from $90^{\circ}$ at off- 
axis viewing directions causes dark-state light leakage which, in turn, degrades the device contrast ratio. By substituting $\phi_{k}=270^{\circ}$ into Eq. (3), the effective polarizer angle $\varphi$ at the lower bisector viewing position $\phi_{0}=270^{\circ}$ can be expressed as:

$$
\varphi=\cos ^{-1}\left(\frac{\sin ^{2} \theta_{0} / n_{p}^{2}}{2-\sin ^{2} \theta_{0} / n_{p}^{2}}\right) .
$$

\subsection{Poincaré Sphere Representation}

For an elliptically polarized light with long axis azimuthal angle $\alpha$ and ellipticity angle $\beta$, its polarization state can be represented by a point $\mathbf{P}$ on the Poincaré sphere with longitude $2 \alpha$ and latitude $2 \beta$ [9], as Fig. 2(a) shows. For a uniaxial film with its optical axis oriented at angle $\gamma$, it can be represented by point $\mathbf{A}$, which is located at longitude $2 \gamma$ on the equator. Suppose this elliptically polarized light (point $\mathbf{P}$ ) passes through the uniaxial film (point A), the overall effect on Poincare sphere is equivalent to rotate AO axis from point $\mathbf{P}$ to point $\mathbf{Q}$ by an angle $\Gamma$, which is determined by the phase retardation of the uniaxial film as expressed in Eqs. (1) and (2). From spherical triangle definition, the spherical angle PAQ is equal to the rotation angle $\Gamma$.

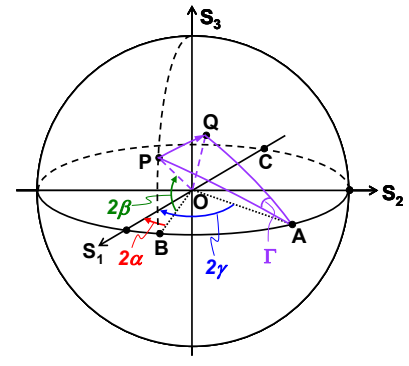

(a)

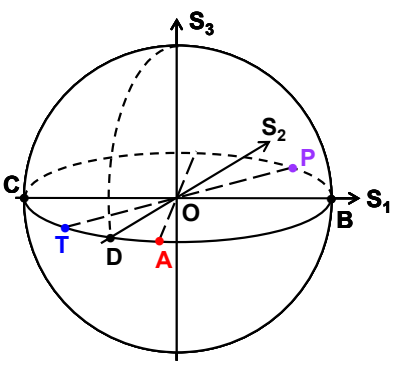

(b)
Fig. 2. Demonstration on the Poincaré sphere about (a) the effect of uniaxial medium on the polarization state change of an incident polarized light, and (b) crossed polarizers under oblique view at the lower bisector viewing position $\phi_{0}=270^{\circ}$ when $\phi_{1}=45^{\circ}$ and $\phi_{2}=-45^{\circ}$, respectively.

The off-axis dark state light leakage of crossed polarizers can also be well explained on the Poincare sphere, as shown in Fig. 2(b). Under oblique view from the lower bisector direction $\phi_{0}=270^{\circ}$, the absorption axis of the polarizer (point $\mathbf{P}$ ) and the absorption axis of the analyzer (point $\mathbf{A}$ ) are not located on the $S_{2}$ axis. Instead, point $\mathbf{P}$ is located between $S_{1}$ and $S_{2}$ axes, while point $\mathbf{A}$ is located between $S_{1}$ and negative $S_{2}$ axes. Moreover, point $\mathbf{P}$ is symmetric to point $\mathbf{A}$ about $S_{1}$ axis, and angle $\angle \mathbf{P O A}$ is twice the effective polarizer angle $\varphi$. Meanwhile, point $\mathbf{T}$, representing the polarization state of light after the polarizer, is on the other end of the diameter passing through point $\mathbf{P}$. Therefore, point $\mathbf{T}$ also deviates from the negative $S_{2}$ axis and is located symmetrically to point A with respect to the negative $S_{2}$ axis. Because point $\mathbf{T}$ does not overlap with point $\mathbf{A}$, light leakage occurs from the bisector viewing direction of the crossed polarizers.

It is easy to figure out the relationship $\angle \mathbf{T O A}=\pi 2 \varphi$ from Fig. 2(b). The larger the angle $\angle$ TOA is, the more severe the light leakage becomes. Since the effective polarizer angle $\varphi$ deviates the farthest from $90^{\circ}$ at all bisector viewing positions, as depicted in Fig. 1(b), the light leakage at bisectors is the severest. If we can suppress the light leakage for all the bisector positions, the viewing angle of the LCD will be significantly enhanced. Thus, the goal of WVA LCDs, which incorporate compensation films into the panel design, is to move point $\mathbf{T}$ to point $\mathbf{A}$ for minimizing the light leakage from the analyzer.

\section{Uniaxial-film compensated IPS-LCD}

As one example to demonstrate how to derive the analytical solutions for uniaxial-film-compensated LCDs, Fig. 3(a) shows the device configuration of an IPS-LCD with one positive a-film and one negative a-film compensation [5]. Figure 3(b) explains the compensation principle on Poincare sphere when the observer views the panel from an oblique angle at the lower bisector position. When the unpolarized light from backlight penetrates the polarizer, whose absorption axis is located at point $\mathbf{P}$, it becomes linearly polarized and its polarization state is located at point $\mathbf{T}$. When such a linearly polarized light (point $\mathbf{T}$ ) passes through the homogenous LC layer, whose position on the Poincaré sphere overlaps with point $\mathbf{P}$, the linear polarization state remains the same (point $\mathbf{T}$ ). Then, the linearly polarized light (point $\mathbf{T}$ ) successively passes through the positive a-film (point A) and the negative a-film (point $\mathbf{P}$ ). When the linearly polarized light (point T) passes through the positive a-film, its polarization state is rotated clockwise from point $\mathbf{T}$ to point $\mathbf{E}$ around the $\mathbf{A O}$ axis. This intermediate polarization state (point $\mathbf{E}$ ), in general, is an elliptical polarization state. By properly choosing the phase retardation values of both positive negative a-films, we can rotate point $\mathbf{E}$ to point $\mathbf{A}$ counterclockwise around the $\mathbf{P O}$ axis. As a result, in the voltage-off state the light is completely absorbed by the analyzer (point A) and a very good dark state is achieved even viewed from an oblique angle at the bisector positions.

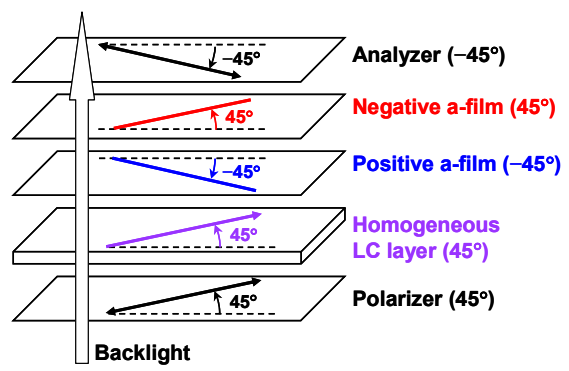

(a)

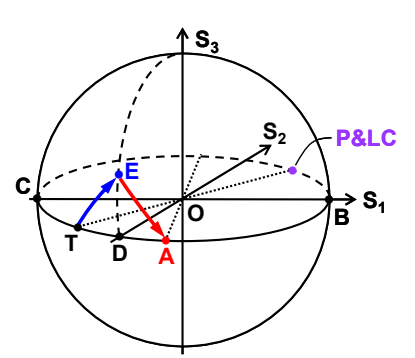

(b)

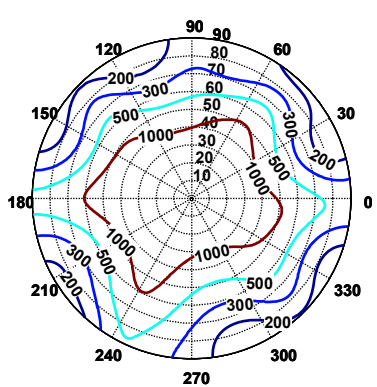

(c)
Fig. 3. (a) Device structure, (b) compensation principle, and (c) viewing angle performance of an IPS-LCD with compensation of one positive a-film and one negative a-film. 
To reach this goal, from Fig. 3(b) we can easily determine the arcs $\overline{\boldsymbol{E A}}=\overline{\boldsymbol{T A}}$ the arcs $\overline{\boldsymbol{T A}}=\overline{\boldsymbol{T} \boldsymbol{E}}$. In other words, the spherical triangle ETA should be an equilateral spherical triangle. Besides, we can also obtain $\angle \mathbf{P O B}=\angle \mathbf{A O B}=\varphi$ and $\overline{\boldsymbol{T A}}=\pi-2 \varphi$ from Fig. 3(b). Based on the spherical trigonometry, we have:

$$
\angle E T A \equiv \angle E A T=\cos ^{-1}(-\operatorname{ctg} \varphi \cdot \operatorname{ctg} 2 \varphi) .
$$

Since the required positive a-film's phase retardation $\Gamma_{\mathrm{a}^{+}}$equals to the spherical angle $\angle \mathbf{E A T}$, thus from Eqs. (1) and (5) the required positive a-film's thickness $d_{\mathrm{a}^{+}}$can be expressed as:

$$
d_{a+}=\lambda \frac{\cos ^{-1}(-\operatorname{ctg} \varphi \cdot \operatorname{ctg} 2 \varphi) / 2 \pi}{n_{a+, e} \sqrt{1-\frac{\sin ^{2} \theta_{0}}{2 n_{a+, e}^{2}}-\frac{\sin ^{2} \theta_{0}}{2 n_{a+, o}^{2}}}-n_{a+, o} \sqrt{1-\frac{\sin ^{2} \theta_{0}}{n_{a+, o}^{2}}}} .
$$

Similarly, the negative a-film's phase retardation $\Gamma_{\mathrm{a}^{-}}$equals to the negative spherical angle $\angle$ ETA, i.e., $\Gamma_{\mathrm{a}}=-\angle \mathbf{E T A}$. Here the minus sign denotes that the phase retardation of the negative afilm is negative and the rotation around $\mathbf{P O}$ axis from point $\mathbf{E}$ to point $\mathbf{A}$ is counterclockwise. Thus, from Eqs. (1) and (5) we can obtain the negative a-film's thickness $d_{\mathrm{a}^{-}}$as:

$$
d_{a-}=-\lambda \frac{\cos ^{-1}(-\operatorname{ctg} \varphi \cdot \operatorname{ctg} 2 \varphi) / 2 \pi}{n_{a-, e} \sqrt{1-\frac{\sin ^{2} \theta_{0}}{2 n_{a-, e}^{2}}-\frac{\sin ^{2} \theta_{0}}{2 n_{a-, o}^{2}}}-n_{a-, o} \sqrt{1-\frac{\sin ^{2} \theta_{0}}{n_{a-, o}^{2}}}} .
$$

From Eqs. (4), (6), and (7), the required film thicknesses depend on the incident angle $\theta_{0}$, film's refractive indices $n_{a^{+}, e}, n_{a^{+}, o}, n_{a^{-}, e}$, and $n_{a-, o}$, and polarizer's average real refractive index $n_{p}$. Therefore, once we know both films' and polarizer's refractive indices as well as the intended viewing angle for LCD optimization, we can determine the required film thickness by using these equations. As an example, if we use the parameters of $n_{L C, e}=1.5649, \quad n_{L C, o}=1.4793, \quad n_{a+, e}=1.5110, \quad n_{a+, o}=1.5095$, $n_{a-, e}=1.5095$, and $n_{a-, o}=1.5110$ and choose $\theta_{0}=70^{\circ}$ as the intended optimization viewing angle, the required film thicknesses as calculated from Eqs. (6) and (7) are $d_{a+}=61.38 \mu \mathrm{m}$ and $d_{a^{-}}=61.37 \mu \mathrm{m}$. Based on these film thicknesses, Fig. 3 (c) plots the calculated iso-contrast contour of a $4-\mu \mathrm{m}$ cell gap IPS-LCD with the compensation of one positive a-film and one negative a-film under white light illumination. We can see that even under white light illumination, the contrast ratio exceeds 100:1 from any viewing angles.

\section{Uniaxial-film compensated MVA-LCD}

As another example to explain the analytical solutions for uniaxial-film-compensated LCDs, Fig. 4(a) shows the schematic device configuration of an MVA-LCD with two positive a-films and two negative c-films [6]. Figure 4(b) explains the compensation principle on Poincaré sphere when the observer views the panel from an oblique angle at the lower bisector position. When the unpolarized light from backlight passes the polarizer (point $\mathbf{P}$ ), it becomes linearly polarized, and its polarization state is located at point $\mathbf{T}$. Such a linearly polarized light (point $\mathbf{T}$ ) then successively goes through the bottom positive a-film (point $\mathbf{A}$ ) and the bottom negative c-film (point $\mathbf{C}$ ). When the linearly polarized light (point $\mathbf{T}$ ) traverses the bottom positive a-film, its polarization state is rotated clockwise around the AO axis and changed from point $\mathbf{T}$ to point $\mathbf{E}$. Point $\mathbf{E}$ is the first intermediate elliptical polarization state. To obtain a symmetric viewing angle, we intentionally let the point $\mathbf{E}$ be located on the great circle passing through $S_{2}$ and $S_{3}$ axes and bisecting the arc $\overline{\boldsymbol{T A}}$. When this elliptically polarized light penetrates the bottom negative c-film, its polarization state is counterclockwise rotated around the $\mathbf{C O}$ axis changing point $\mathbf{E}$ to point $\mathbf{F}$. Point $\mathbf{F}$ is the second intermediate elliptical polarization state, which is also located on the same great circle as point E. Afterwards, this second intermediate elliptically polarized light passes through the unactivated MVA LC layer (point $\mathbf{C}$ ), and its polarization state is clockwise rotated around the $\mathbf{C O}$ axis moving point $\mathbf{F}$ to point $\mathbf{G}$. Point $\mathbf{G}$ is the third intermediate elliptical polarization state, which again is located on the same great circle as points $\mathbf{E}$ and $\mathbf{F}$. Now this third intermediate elliptically polarized light (point G) successively passes through the top negative c-film (point $\mathbf{C}$ ) and the top positive a-film (point $\mathbf{P}$ ). When the third intermediate polarized light passes through the top negative c-film, its polarization state is rotated counterclockwise around the $\mathbf{C O}$ axis changing point $\mathbf{G}$ to point $\mathbf{H}$. Point $\mathbf{H}$ is the fourth intermediate elliptical polarization state, which is once again located on the same great circle as points $\mathbf{E}, \mathbf{F}$, and $\mathbf{G}$. When the fourth intermediate elliptically polarized light passes through the top positive a-film, its polarization state can be rotated clockwise around the $\mathbf{P O}$ axis moving point $\mathbf{H}$ to point $\mathbf{A}$, provided that we properly design the phase retardation values of all four uniaxial films. As a result, at the voltage-off state the light is completely absorbed by the analyzer (point $\mathbf{A}$ ) resulting in a good dark state even if it is viewed from the bisector directions.

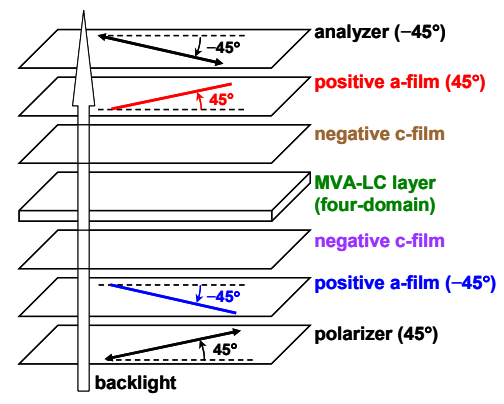

(a)

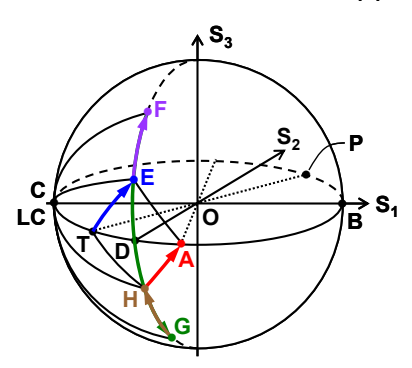

(b)

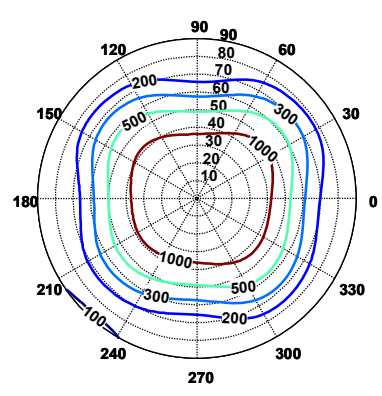

(c)
Fig. 4. (a) Device structure, (b) compensation principle, and (c) viewing angle performance of an MVA-LCD with compensation of two positive a-films and two negative c-films.

To keep symmetric viewing angle all the four intermediate polarization states must be located on the great circle passing through $S_{2}$ and $S_{3}$ axes and bisecting the arc $\overline{\boldsymbol{T A}}$. Moreover, point $\mathbf{E}$ and point $\mathbf{H}$ should be symmetric to each other with respect to 
the equator plane, so should point $\mathbf{F}$ and point $\mathbf{G}$. From the Poincaré sphere, we find that the $\operatorname{arcs} \overline{\boldsymbol{E T}}, \overline{\boldsymbol{E A}}, \overline{\boldsymbol{T A}}, \overline{\boldsymbol{H T}}$, and $\overline{\boldsymbol{H A}}$ all should be equal to each other. In other words, both spherical triangles ETA and HTA are equilateral spherical triangles. In addition, we can also obtain $\overline{\boldsymbol{C F}}=\overline{\boldsymbol{C E}}=\overline{\boldsymbol{C D}}=\overline{\boldsymbol{C H}}$ $=\overline{\boldsymbol{C G}}=\pi 2, \angle \mathbf{P O B}=\angle \mathbf{A O B}=\varphi$, and $\overline{\boldsymbol{T A}}=\pi-2 \varphi$ from Fig. 4(b). Based on spherical trigonometry, we have:

$$
\begin{aligned}
& \angle E A T=\angle A T H=\cos ^{-1}(-\operatorname{ctg} \varphi \cdot \operatorname{ctg} 2 \varphi), \\
& \angle E C D=\angle H C D=\cos ^{-1}(-\cos 2 \varphi / \sin \varphi), \\
& \angle F C D=\angle G C D=\Gamma_{L C} / 2,
\end{aligned}
$$

where $\Gamma_{L C}$ is the phase retardation introduced by the unactivated MVA LC layer for an oblique incident light:

$$
\Gamma_{L C}=\frac{2 \pi}{\lambda} n_{L C, o} d_{L C}\left(\sqrt{1-\frac{\sin ^{2} \theta_{0}}{n_{L C, e}^{2}}}-\sqrt{1-\frac{\sin ^{2} \theta_{0}}{n_{L C, o}^{2}}}\right) .
$$

To calculate the thickness of each compensation film, the following information is useful. The bottom positive a-film's phase retardation is equal to the spherical angle $\angle \mathbf{E A T}$, while the top positive a-film's phase retardation equals to the spherical angle $\angle \mathbf{A T H}$. Thus, the required film thicknesses for both positive a-films should be equal to each other, as indicated by Eq. (8a). From Eqs. (1) and (8a), the required film thickness $d_{a^{+}}$for both positive a-films is:

$$
d_{a+}=\lambda \frac{\cos ^{-1}(-\operatorname{ctg} \varphi \cdot \operatorname{ctg} 2 \varphi) / 2 \pi}{n_{a+, e} \sqrt{1-\frac{\sin ^{2} \theta_{0}}{2 n_{a+, e}^{2}}-\frac{\sin ^{2} \theta_{0}}{2 n_{a+, o}^{2}}}-n_{a+, o} \sqrt{1-\frac{\sin ^{2} \theta_{0}}{n_{a+, o}^{2}}}} .
$$

On the other hand, from Fig. 4(b), the required bottom negative cfilm's phase retardation equals to the negative spherical angle $\angle \mathbf{E C F}$, while the required top negative c-film's phase retardation equals to the negative spherical angle $\angle$ HCG. As a result, the required film thickness for both negative c-films should equal to each other, as indicated by Eqs. (8b) and (9c). Accordingly, the required phase retardation for both negative c-films is $\Gamma_{c^{-}}=-\angle \mathbf{E C F}=-\angle \mathbf{H C G}=\angle \mathbf{E C D}-\angle \mathbf{F C D}=\angle \mathbf{H C D}-\angle \mathbf{G C D}$. Thus from Eqs. (2), (8b), and (8c), the required film thickness $d_{c^{-}}$for both negative c-films has the following expression:

$$
d_{c-}=\lambda \frac{\left[\cos ^{-1}(-\cos 2 \varphi / \sin \varphi)-\Gamma_{L C} / 2\right] / 2 \pi}{n_{c-, o}\left(\sqrt{1-\frac{\sin ^{2} \theta_{0}}{n_{c-, e}^{2}}}-\sqrt{1-\frac{\sin ^{2} \theta_{0}}{n_{c-, o}^{2}}}\right)} .
$$

As seen from Eqs. (9)-(11), once we know both films' and polarizer's refractive indices as well as the intended viewing angle for optimizing the LCD panel, we can determine the required compensation films' thickness from Eqs. (9)-(11). For instance, if we use the parameters of $n_{L C, e}=1.5606, \quad n_{L C, o}=1.4770$, $n_{a+, e}=1.5110, n_{a^{+}, o}=1.5095, n_{c^{-}, e}=1.5095$, and $n_{c^{-}, o}=1.5110$ and choose $\theta_{0}=70^{\circ}$ as the intended optimization viewing angle, the required film thicknesses are $d_{a^{+}}=61.38 \mu \mathrm{m}$ and $d_{c^{-}}=72.14 \mu \mathrm{m}$. Using these film thicknesses, Fig. 4 (c) plots the calculated isocontrast contour of a $4.6-\mu \mathrm{m}$ cell gap MVA-LCD with the compensation of two positive a-films and two negative c-films under white light illumination. Again, we can see that even under white light illumination, the contrast ratio is still over 100:1 from any viewing angles.

\section{Conclusion}

For the first time, we systematically derive a set of analytical solutions for the uniaxial-film compensated LCDs. These analytical solutions not only provide a straightforward method to obtain the required compensation film's parameters but also clearly explain the physical principle by the means of Poincaré sphere representation. The analysis process can be applied to most of the mainstream uniaxial-film compensated wide-view LCDs [10]. Similar analyses on biaxial film compensated wide-view LCDs are underway. Its significant impact to LCD industry is foreseeable.

\section{Acknowledgement}

This work is supported by Toppoly Optoelectronics Corp. (Taiwan).

\section{References}

[1]. M. Oh-e, M. Ohta, S. Aratani, and K. Kondo, Proc. 15th Int'l Display Research Conference (Asia Display'95), 577 (1995).

[2]. A. Takeda, S. Kataoka, T. Sasaki, H. Chida, H. Tsuda, K. Ohmuro, Y. Koike, T. Sasabayashi, and K. Okamoto, SID Digest Tech. Papers, 29, 1077 (1998).

[3]. J. Chen, K. -H. Kim, J. -J. Jyu, J. H. Souk, J. R. Kelly, and P. J. Bos, SID Digest Tech. Papers, 29, 315 (1998).

[4]. J. E. Anderson and P. J. Bos, Jpn. J. Appl. Phys, Pt.1, $\underline{39}$, 6388 (2000).

[5]. X. Zhu and S. T. Wu, SID Digest Tech. Papers, 34, 1164 (2005).

[6]. Q. Hong, T. X. Wu, X. Zhu, R. Lu, and S. -T. Wu, Appl. Phys. Lett., $\underline{86}, 121107$ (2005).

[7]. Y. Saitoh, S. Kimura, K. Kusafuka, and H. Shimizu, Jpn. J. Appl. Phys., Pt.1, 37, 4822 (1998).

[8]. T. Ishinabe, T. Miyashita, T. Uchida, and Y. Fujimura, Proc. 21st Int'1 Display Research Conference (Asia Display/IDW'01), 485 (2001).

[9]. J. E. Bigelow and R. A. Kashnow, Appl. Opt. 16, 2090 (1977).

[10]. X. Zhu, Z. Ge, and S. T. Wu, IEEE/OSA J. Display Technology, 2, 2 (2006). 